\title{
Severe symptomatic hyponatremia associated with the use of polyethylene glycol-based bowel preparation
}

\author{
Navira Samad and Ian Fraser \\ Epworth HealthCare, Melbourne, Victoria, Australia
}

Correspondence

should be addressed

to N Samad

Email

navira7@hotmail.com

\section{Summary}

Colonoscopy is a useful tool in modern medicine and is increasingly employed for both diagnostic and treatment reasons. However, its effectiveness is highly reliant on the quality of bowel cleansing. Among different bowel-cleansing agents available, PEG (polyethylene glycol) is considered to be the safest cleansing agent, especially in relation to fluid and electrolyte problems. We present here a case of severe symptomatic hyponatremia that developed after the use of PEG for an elective colonoscopy. This case highlights that despite the use of PEG-based preparations, life-threatening fluid and electrolyte disturbances can still occur in patients with risk factors, such as old age, use of thiazide diuretics and SSRIs, chronic kidney disease, heart failure and a history of electrolyte problems. These patients should be closely monitored when undertaking bowel cleansing and should receive prompt care in the event of complications, to avoid permanent neurological sequelae and death. Rapid correction of sodium levels in patients requiring treatment of hyponatremia should be avoided to prevent complications such as osmotic demyelination syndrome.

\section{Learning points:}

- PEG is considered to be the safest bowel-cleansing agents among different options available, but it can still cause significant side effects in susceptible individuals.

- Those at risk of developing adverse events include elderly individuals, patients with chronic kidney disease, heart failure or previous history of electrolyte problems and those taking thiazide diuretics and SSRIs.

- All such patients should be closely monitored i.e. have their metabolic profile checked prior to the commencement of bowel cleansing and a low threshold should be kept for the initiation of investigations and treatment in case of development of symptoms.

- Medications with a potential of causing fluid and electrolytes such as thiazide diuretics and SSRIs should be withheld while patient is undertaking bowel preparation.

- Hyponatremia in a hospitalized patient can be multifactorial, and the treatment principles are based on duration of onset, presence of symptoms and patients volume status.

- Overzealous correction of sodium levels during treatment of hyponatremia can result in serious complications such as osmotic demyelination syndrome.

\section{Background}

Population-based screening for bowel cancer is performed using the fecal occult blood test (FOBT). Colonoscopy is the recommended follow-up test for those with positive findings on FOBT screening and as a primary tool for cancer surveillance in people with an increased risk of bowel cancer, such as those with a first-degree relative

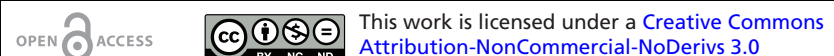
Unported License.
} $\begin{array}{rr}\text { C } 2017 \text { The authors } & \text { http://www.edmcasereports.com } \\ \text { Published by Bioscientifica Ltd }\end{array}$ 
who was diagnosed with bowel cancer under the age of 55 years (1) (see reference for Cancer Council). Moreover, endoscopic surveillance for colorectal cancers has been shown to significantly reduce mortality (see reference Cancer Council Australia). An excellent bowel preparation is critical for a colonoscopy (2) because it permits the visualization of the entire colonic mucosa and increases the safety of therapeutic maneuvers (3). Two main types of bowel preparations are available including hyperosmotic and iso-osmotic preparations, combined with stimulant laxatives to maximize the bowel-cleansing effect (3).

Hyperosmotic formulations include sodium phosphate, sodium sulfate, sodium pico sulfate and magnesium citrate. However, these are usually avoided because of concerns over their safety and their potential to cause fluid and electrolyte problems and renal impairment. PEG-based preparations are iso-osmotic and have a better safety profile, as they are minimally absorbed in the gastrointestinal tract (4). Although they are the preferred agents of choice worldwide, there have been documented case reports of adverse events including seizures, renal impairment, upper gastrointestinal bleeding, cardiac events and death $(5,6)$. Here, we report a case of a 68 -year-old female presenting with generalized tonicclonic seizures and hyponatremia after administration of a PEG-based preparation for bowel cleansing as per the recommended protocol.

\section{Case presentation}

A 68-year-old lady was admitted to ICU for the management of seizures due to hyponatremia. She had a medical history of breast cancer with a recurrence in 2011 requiring partial right-sided mastectomy, adjuvant radiotherapy and hormone therapy. She also had a history of right-sided nephrectomy, esophageal dysmotility, hypertension, tonsillectomy and a previous episode of colonic bleeding. Her regular medications included irbesartan, hydrochlorothiazide, tamoxifen, rabeprazole, atorvastatin, vitamin $\mathrm{D}$ and ezetimibe.

She had been feeling unwell and nauseous after a couple of hours of commencing bowel cleansing. She took two doses of Pico Prep and one dose of colonlytely (PEG) according to the instructions. She later developed profuse vomiting and had to cancel the colonoscopy due to ongoing nausea, vomiting and malaise. Her symptoms continued for two days during which she had minimal oral intake. She subsequently developed a generalized tonic-clonic seizure culminating in her presentation to the emergency department. Before commencing bowel cleansing, she had been perfectly well and took her prescription medications as usual.

On arrival, her vital signs were stable with a blood pressure of $150 / 70 \mathrm{mmHg}$, pulse rate of 84 beats per min and oxygen saturation of $98 \%$ on room air. On physical examination, she appeared confused but had no focal neurological deficits. She had normal skin turgor and moist mucous membranes, with a normal cardiorespiratory and abdominal examination.

\section{Investigation}

Initial investigations showed severe hyponatremia with a serum $\mathrm{Na}+$ of $106 \mathrm{mmol} / \mathrm{L}$ (135-145), mild hypokalemia with $\mathrm{K}+$ of $3.1 \mathrm{mmol} / \mathrm{L}(3.5-5.5)$ and a reduced serum osmolality of $224 \mathrm{mosmol} / \mathrm{kg}$ (280-300). The urine osmolality was $525 \mathrm{mosmol} / \mathrm{kg}(50-1200)$ and the urine $\mathrm{Na}+$ was $74 \mathrm{mmol} / \mathrm{L}$ (40-300). She had normal kidney function, and no evidence of thyroid or adrenal insufficiency. Imaging revealed a normal chest X-ray and CT brain.

\section{Treatment}

She was admitted to intensive care unit for management of severe symptomatic hyponatremia. She was initially treated with 3\% normal saline, and the infusion rates were adjusted according to the 2 hourly electrolyte checks with target sodium increments of $1-2 \mathrm{mmol} / \mathrm{L} / \mathrm{h}$. The sodium levels increased to $112 \mathrm{mmol} / \mathrm{L}$ after $6 \mathrm{~h}$; hence, the hypertonic saline infusion was ceased and she was placed on fluid restriction. Subsequently, the sodium levels gradually increased to $118 \mathrm{mmol} / \mathrm{L}$ and $125 \mathrm{mmol} / \mathrm{L}$ after 36 and $48 \mathrm{~h}$ respectively. Her mental status improved during this time, and she did not have any further seizures. The sodium levels normalized after 5 days and remained stable until discharge. Her antihypertensive medications were ceased during the admission.

\section{Outcome and follow-up}

She made a full recovery without any permanent neurological damage.

One-month follow-up showed normal serum sodium levels; however, her blood pressure was elevated, therefore, irbesartan was recommenced. 


\section{Discussion}

Hyponatremia is one of the most common electrolyte disorders encountered in clinical practice with a reported incidence of $15-30 \%$ (7). Clinical manifestations directly attributable to hyponatremia depend on its duration and severity. They are primarily neurologic and reflect the changes in cerebral hydration in response to changing serum sodium concentration. Symptoms can range from subtle confusion, gait disturbances and attention deficit in chronic cases to seizures coma and obtundation in the acute hyponatremia. It is often multifactorial, and multiple aetiologies can be found in an individual patient, which can sometimes present a diagnostic and management dilemma to the clinicians.

The general approach for treating patients with hyponatremia depends on the duration and severity of hyponatremia and the presence of symptoms. Patient receiving intravenous fluids for severe hyponatremia should be closely monitored to avoid overzealous correction of sodium, as this can lead to 'osmotic demyelination syndrome'. It is generally agreed that the correction of sodium should not exceed $1-2 \mathrm{mmol} / \mathrm{h}$ and $8 \mathrm{mmol} / \mathrm{day}$ on any given day of treatment. The target should not be to normalize serum sodium but to raise it to safe levels $(>120 \mathrm{mmol} / \mathrm{L})$ after which conservative measures such as fluid restriction can be deployed (7). Patients with chronic hyponatremia are at even higher risk of osmotic demyelination due to brain adaptation, which can even develop with water restriction alone; hence, sodium correction in these patients should be very slow and gradual.

Hyponatremia in our patient was most likely the result of large volume loss and diuretic use combined with physiological non-osmotic ADH response, in an older patient. The sodium increments throughout her treatment remained within the recommended range i.e. $6-8 \mathrm{mmol} / \mathrm{L} /$ day, and she did not suffer from any complications of osmotic demyelination.

$\mathrm{ADH}$ secretion is regulated primarily by changes in osmolarity and tonicity. Other non-osmotic signals such as stress, pain, hypoglycemia, nausea and manipulation of abdominal contents also stimulate ADH secretion (8).

$\mathrm{ADH}$ release helps reabsorption of water by acting through v2 receptors on the basolateral membrane of distal convoluted tubules and collecting ducts. Binding of $\mathrm{ADH}$ to these receptors stimulates the recruitment of selective water channels (aquaporins), which allow reabsorption of renal tubular water and concentration of urine (8).
In our patient, $\mathrm{ADH}$ release was stimulated by two mechanisms, including acute loss of fluid leading to hypovolemia and hyperosmolarity and secondly by ongoing vomiting and nausea.

She was also taking thiazide diuretics, which further compounded the problem. Thiazides block reabsorption of sodium from the distal tubules and enhance water retention by both $\mathrm{ADH}$-dependent and $\mathrm{ADH}$-independent mechanisms (9). The combination of increased sodium and potassium excretion (due to the diuretic) and enhanced water reabsorption can result in the excretion of urine with a sodium plus potassium concentration higher than that of the plasma. Loss of this fluid can directly promote the development of hyponatremia independent of the degree of water intake (9).

Another differential diagnosis, in this case, was SIADH, which occurs if ADH secretion is not suppressed when plasma sodium concentration falls below the osmotic threshold for ADH release (8). SIADH can be secondary to a variety of problems, including malignancies, drugs, CNS disturbances, pulmonary disease, and sometimes, it may be multifactorial (8). Bartter and Schwartz criteria for diagnosis of SIADH include a decreased plasma osmolality of less than $275 \mathrm{mosmol} / \mathrm{kg}$, inappropriately concentrated urine ( $>100 \mathrm{mosmol} / \mathrm{kg}$ ), a euvolemic state, elevated urine $\mathrm{Na}(>20 \mathrm{mEq} / \mathrm{L})$, the absence of diuretic use and thyroid or adrenal insufficiency (10). Because of her history of cancers, we ordered an imaging of chest, abdomen and pelvis to look for a paraneoplastic source of ectopic $\mathrm{ADH}$ that may have resulted in SIADH. However, we could not find any evidence. Although the urine sodium concentration in this case was above $30 \mathrm{mmol} / \mathrm{L}$, diagnosis of SIADH was excluded due to the use of diuretics.

There have been previous case reports of hyponatremia, associated with PEG intake, which have resulted in serious adverse events requiring hospitalization (Table 1). Most of the patients were over 60 years of age, and only two were in their 50s. There was a female preponderance; 7 patients were female and only one patient was male. The majority of patients had known risk factors for hyponatremia; one patient (\#7) had chronic kidney disease with defective free water clearance. Three patients (\#1, \#2 and \#8) were taking thiazides, which would have impaired water excretion. One patient (\#6) was taking SSRIs and had inadequate thyroxine replacement. Patients \#4, \#5 and \#9 did not have prior risk factors, and the most likely mechanism of hyponatremia in these patients was significant volume loss and physiological non-osmolar antidiuretic hormone stimulation. Hyponatremia patient \#3 was 


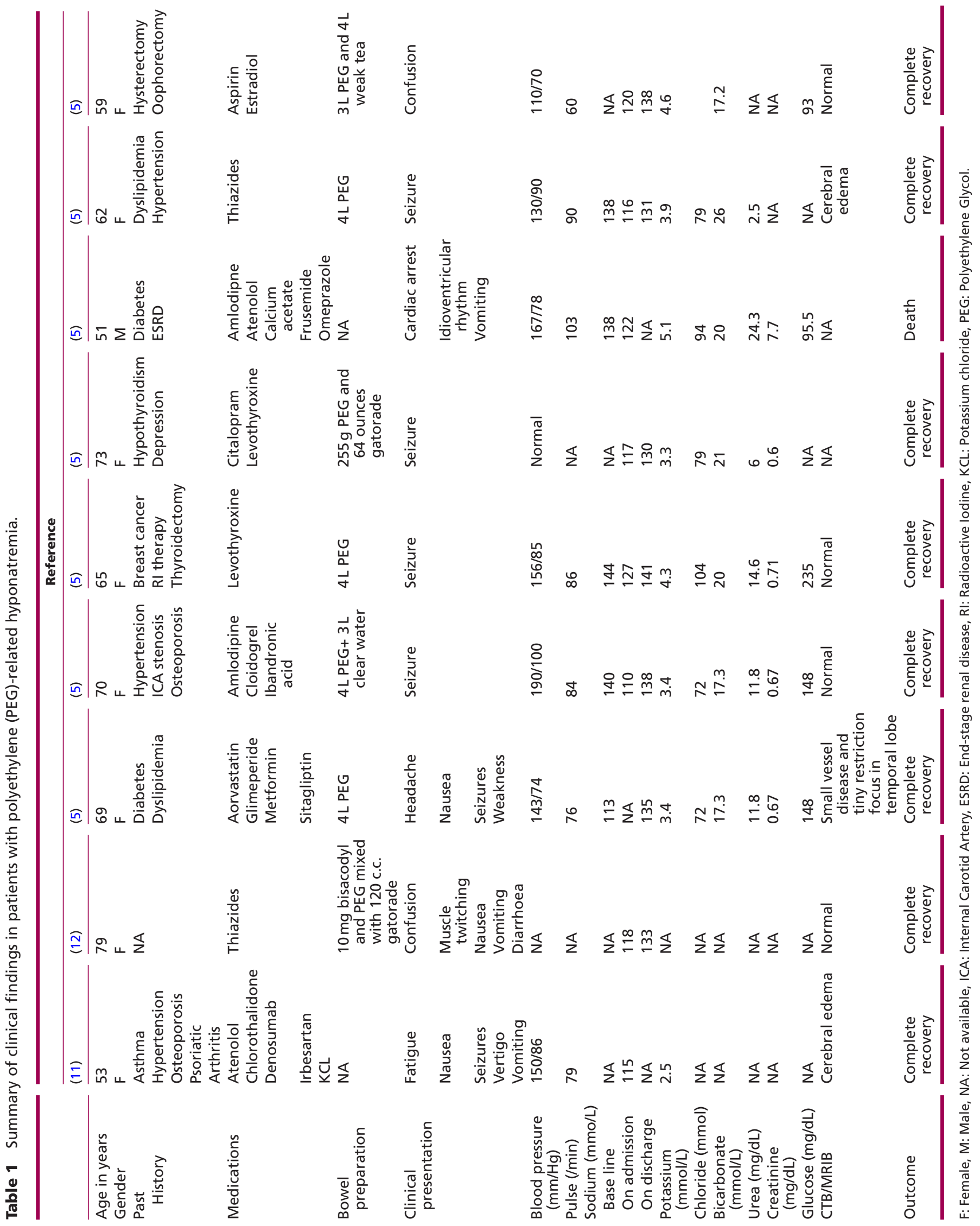


attributed to newly developed SIADH, resulting from PEG use. All patients had complete recovery except for 1 patient who had dialysis-dependent CKD and died as a result of cardiac arrest $(5,11,12)$.

\section{Declaration of interest}

The authors declare that there is no conflict of interest that could be perceived as prejudicing the impartiality of the research reported.

\section{Funding}

This research did not receive any specific grant from any funding agency in the public, commercial or not-for-profit sector.

\section{Patient consent}

A written informed consent has been obtained from the patient for publication of the submitted article.

\section{Author contribution statement}

Navira Samad (70\%): Literature review and drafting of the paper; read and approved the final version of the paper to be published. Ian Fraser (30\%): Critical review and editing of the manuscript for intellectual content; read and approved the final version of the paper to be published.

\section{References}

1 Cancer Council Australia Colonoscopy Surveillance Working Party 2011 Clinical Practice Guidelines for Surveillance Colonoscopy - in adenoma follow-up; following curative resection of colorectal cancer and for cancer surveillance in inflammatory bowel disease. Sydney:
Cancer Council Australia; (cited 2016 Oct). (Available from: https:// www.nhmrc.gov.au/_files_nhmrc/publications/attachments/ext0008_ colonoscopy_guideline_120314.pdf)

2 Rex DK, Petrini JL, Baron TH, Chak A, Cohen J, Deal SE, Hoffman B, Jacobson BC, Mergener K, Petersen BT, et al. 2015 Quality indicators for colonoscopy. Gastrointestinal Endoscopy 81 31-53. (doi:10.1016/j. gie.2014.07.058)

3 Zauber AG, Winawer SJ, O’Brien MJ, Lansdorp-Vogelaar I, van Ballegooijen M, Hankey BF, Shi W, Bond JH, Schapiro M, Panish JF, et al. 2012 Colonoscopic polypectomy and long-term prevention of colorectal-cancer deaths. New England Journal of Medicine 366 687-696. (doi:10.1056/NEJMoa1100370)

4 Moon W 2013 Optimal and safe bowel preparation for colonoscopy. Clinical Endoscopy 46 219-223. (doi:10.5946/ce.2013.46.3.219)

5 Ko SH, Lim CH, Kim JY, Kang SH, Baeg MK \& Oh HJ 2014 Case of inappropriate $\mathrm{ADH}$ syndrome: hyponatremia due to polyethylene glycol bowel preparation. World Journal of Gastroenterology 20 12350-12354. (doi:10.3748/wjg.v20.i34.12350)

6 Hasan AG \& Brown WR 2011 Colonic cleansing for colonoscopy: a risk to be taken seriously. Gastrointestinal Endoscopy 73 616-618. (doi:10.1016/j.gie.2010.11.050)

7 Biswas M \& Davies JS 2007 Hyponatraemia in clinical practice. Postgraduate Medical Journal 83 373-378. (doi:10.1136/ pgmj.2006.056515)

8 Hannon MJ \& Thompson CJ 2016 Endocrinology: Adult and Pediatric; Cellular Action of Vasopressin, 7th ed., Vol. 1. N.P.: Saunders.

9 Cesar KR \& Magaldi AJ 1999 Thiazide induces water absorption in the inner medullary collecting duct of normal and Brattleboro rats. American Journal of Physiology 277 F756-F760.

10 Bartter FC \& Schwartz WB 1967 The syndrome of inappropriate secretion of antidiuretic hormone. American Journal of Medicine $\mathbf{4 2}$ 790-806. (doi:10.1016/0002-9343(67)90096-4)

11 Brousseau SB 2015 Seizure associated with hyponatremia possibly related to the use of polyethylene glycol and electrolytes preparation. Journal of Clinical Toxicology 229.

12 Pazderka P \& Hollensteiner C 2015 Severe Hyponatremic Encephalopathy Following Bowel Prep for Colonoscopy. Emergency Physicians.

Received in final form 12 December 2016

Accepted 19 January 2017 